\title{
Efeitos da temperatura e da movimentação do ar sobre o isolamento térmico do velo de ovinos em câmara climática
}

\author{
Alex Sandro Campos Maia ${ }^{1}$, Roberto Gomes da Silva², Patrícia de Cássia Andrade ${ }^{3}$ \\ ${ }^{1}$ Laboratório de Biometeorologia e Bem-Estar Animal, Departamento de Ciências Animais da Universidade Federal Rural do Semi-Árido, \\ Mossoró, Rio Grande do Norte. \\ ${ }^{2}$ Departamento de Zootecnia da Faculdade de Ciências Agrárias e Veterinária, Universidade Estadual Paulista, Campus de Jaboticabal, \\ Jaboticabal, São Paulo. \\ ${ }^{3}$ Curso de Pós-graduação em Zootecnia da Faculdade de Ciências Agrárias e Veterinária, Universidade Estadual Paulista, Campus de \\ Jaboticabal, Jaboticabal, São Paulo.
}

RESUMO - Foram utilizados dez ovinos da raça Corriedale - cinco machos e cinco fêmeas com pesos entre 52,2 e $87,6 \mathrm{~kg}$ - com o objetivo de avaliar o efeito da combinação da movimentação do ar (0 e 5,0 m/s) com a temperatura do ar $\left(25,30\right.$ e $\left.40^{\circ} \mathrm{C}\right)$ sobre a temperatura retal $\left(\mathrm{T}_{\mathrm{R}},{ }^{\circ} \mathrm{C}\right)$, da epiderme $\left(\mathrm{T}_{\mathrm{E}},{ }^{\circ} \mathrm{C}\right)$, da superfície do velo $\left(\mathrm{T}_{\mathrm{V}}\right.$, $\left.{ }^{\circ} \mathrm{C}\right)$ e do interior do velo $\left(\mathrm{T}_{\mathrm{I}},{ }^{\circ} \mathrm{C}\right)$ e a espessura do velo $\left(\mathrm{E}_{\mathrm{V}}, \mathrm{cm}\right)$ e suas relações com o isolamento térmico do velo. A presença de vento não teve efeito sobre as variáveis estudadas, o que sugere que fluxo de ar $(<5,0 \mathrm{~m} / \mathrm{s})$ paralelo ao eixo corporal do animal tem pouco efeito sobre o isolamento térmico do velo, independentemente da temperatura do ar, que se mostrou altamente correlacionada, de forma positiva, com as temperaturas retal, do velo, do interior do velo e da epiderme. Sob temperaturas inferiores a $30^{\circ} \mathrm{C}$, a transferência de calor através do velo ocorreu via condução e convecção livre, enquanto sob altas temperaturas $\left(>40^{\circ} \mathrm{C}\right)$ o fluxo de calor sensível não foi significativa.

Palavras-chave: vento, calor sensível, ovinos

\section{Effect of temperature and air velocity on the thermal insulation of the fleece of sheep in climatic chamber}

\begin{abstract}
Ten Corriedale sheep were evaluated, five males and five females with 52.2 to $87.6 \mathrm{~kg}$ of body weight, to evaluate the effect of air velocity $(0$ and $5 \mathrm{~m} / \mathrm{s})$ and temperature $\left(25,30\right.$ and $\left.40^{\circ} \mathrm{C}\right)$ on rectal temperature $\left(\mathrm{T}_{\mathrm{R}}\right.$, $\left.{ }^{\circ} \mathrm{C}\right)$, skin temperature $\left(\mathrm{T}_{\mathrm{E}},{ }^{\circ} \mathrm{C}\right)$, fleece surface temperature $\left(\mathrm{T}_{\mathrm{V}},{ }^{\circ} \mathrm{C}\right)$, temperature inside the fleece $\left(\mathrm{T}_{\mathrm{I}},{ }^{\circ} \mathrm{C}\right)$, and fleece thickness $\left(E_{V}, c m\right)$ and their relationship with fleece thermal insulation. The results showed that air velocity did not affect the studied traits, suggesting that airflow $<5.0 \mathrm{~m} / \mathrm{s}$ parallel to the fleece had little effect on fleece thermal insulation, regardless the air temperature. Air temperature was highly correlated with $\mathrm{T}_{\mathrm{R}}, \mathrm{T}_{\mathrm{E}}, \mathrm{T}_{\mathrm{I}}$ and $\mathrm{T}_{\mathrm{V}}$. Under air temperatures below $30^{\circ} \mathrm{C}$, the heat transfer through the fleece was dominated by conduction and free convection, while under high temperatures $\left(>40^{\circ} \mathrm{C}\right)$, the sensible heat flow was not significant.
\end{abstract}

Key Words: wind, sensible heat, sheep

\section{Introdução}

A habilidade de um animal homeotérmico em manter sua temperatura corporal praticamente constante sob condições de alta temperatura ambiente está diretamente relacionada à sua eficiência em perder calor através da superfície corporal (Gebremedhin et al., 1983; McArthur, 1987). Na ausência de uma capa de cobertura espessa, o calor é perdido diretamente da superfície cutânea para o ambiente, o que não ocorre em superfície coberta por uma capa, na qual a troca de calor do corpo com o ambiente é determinada pelo isolamento térmico proporcionado pela capa (Davis \& Birkebak, 1974; Cena \& Monteith, 1975abc; Ehrlemark \& Sallvik, 1996; Berman, 2004). Cada tipo de cobertura adiciona grau de isolamento térmico diferente (Blaxter et al., 1959; Treggear, 1965; Walsberg et al., 1978).

Esse isolamento é bidirecional, isto é, constitui uma barreira à passagem da energia térmica procedente do ambiente externo como do próprio organismo (Whates \& Charles, 1994). A eficiência do isolamento depende basicamente dos processos que determinam a transferência de calor da superfície da epiderme do animal através do seu 
pelame para a vizinhança. Segundo Cena \& Monteith (1975b), essa transferência sensível é dominada, no caso de uma atmosfera imóvel, pela convecção livre associada a diferentes densidades do ar dentro do pelame, pela troca radiativa entre os pêlos e pela condução ao longo desses pêlos.

Quando um animal é exposto a uma atmosfera imóvel, o valor médio do isolamento térmico da capa varia entre 0,074 e $0,270 \mathrm{~m}^{2} /{ }^{\circ} \mathrm{K} / \mathrm{W}$ a cada cm de espessura da capa, dependendo da espécie (Monteith \& Unsworth, 1990). No caso de haver movimentação do ar, e se este ar penetrar no interior do velo, poderá reduzir consideravelmente o isolamento térmico (McArthur \& Monteith, 1980), principalmente em pelames menos densos (Treggear, 1965) ou em velos menos espessos (Cena \& Monteith, 1975b). Obviamente, em uma atmosfera não-imóvel, a convecção forçada domina o processo de transferência de calor através da capa, dependendo do nível de movimentação do ar e do quanto esse ar penetra no interior do velo (Cena \& Monteith, 1975b).

Assim, torna-se importante o conhecimento da interação entre os fatores ambientais, neste caso a movimentação do ar, sobre o isolamento térmico em ovinos lanados aclimatados às condições adversas do ambiente tropical, uma vez que, em toda literatura revisada, os dados foram obtidos sob altas latitudes ou a partir de amostras de pele. Por isso, neste trabalho investigou-se o efeito do aumento da temperatura do ar, combinada com a movimentação do ar, sobre as temperaturas retal, da superfície da epiderme, do interior do velo e da superfície do velo e suas relações com o isolamento térmico do velo de ovinos.

\section{Material e Métodos}

O trabalho foi conduzido na câmara climática do Laboratório de Bioclimatologia da Faculdade de Ciências Agrárias e Veterinárias da Universidade Estadual Paulista (UNESP), Campus de Jaboticabal, São Paulo. A partir de um rebanho com 23 ovinos da raça Corriedale, constituído de indivíduos adultos, borregos e recém-nascidos, foram selecionados aleatoriamente dez animais adultos (cinco machos e cinco fêmeas pesando entre 52,2 e 87,6 kg). Em cada sessão de observação, os dez animais foram colocados no interior da câmara climática e avaliados sob três níveis de temperatura do $\operatorname{ar}\left(T_{A}=25,30\right.$ e $\left.40^{\circ} \mathrm{C}\right)$ combinados com dois níveis de movimentação do ar ( $V=0$ e $5,0 \mathrm{~m} / \mathrm{s}$ ), perfazendo um total de 60 observações por dia. Esses animais recebiam água à vontade, feno de coast-cross picado (Cynodon dactylon L. Pers), silagem de milho e concentrado comercial misturados na proporção de 30, 60 e $10 \%$, respectivamente. O concentrado era composto de $93 \%$ de farelo de trigo e $7 \%$ de farelo de soja. Cada sessão iniciava às $8 \mathrm{~h}$ e terminava por volta das $18 \mathrm{~h}$, com cinco repetições para cada temperatura e movimentação do ar, entre julho e setembro de 2003. Durante esse período, os animais selecionados não foram tosquiados.

A movimentação do ar sobre os animais foi promovida por ventiladores instalados de modo que o fluxo de ar, medido com termo-anemômetro Alnor APM-360, fosse paralelo ao eixo corporal. Realizaram-se as seguintes medições: espessura do velo $\left(E_{V}, \mathrm{~cm}\right)$, medida com paquímetro digital; temperatura da superfície do velo $\left(T_{V},{ }^{\circ} \mathrm{C}\right)$ e temperatura da superfície da epiderme $\left(T_{E},{ }^{\circ} \mathrm{C}\right)$, medidas com termômetro infravermelho (Rynger ${ }^{\circledR} \mathrm{ST}^{\mathrm{TM}}$ ); temperatura retal $\left(T_{R},{ }^{\circ} \mathrm{C}\right)$, com termômetro clínico; e temperatura interna do velo $\left(T_{I},{ }^{\circ} \mathrm{C}\right)$, com a ponta-de-prova conectada ao termômetro infravermelho, inserida até o centro do velo par leitura, realizada após 3 minutos.

Os dados foram analisados pelo método dos quadrados mínimos usando o programa "Statistical Analyses System" (SAS, 2001), conforme descrito por Littell et al. (1991). O modelo matemático utilizado foi:

$$
\begin{gathered}
\mathrm{Y}_{\mathrm{ijklmn}}=\alpha+\mathrm{s}_{\mathrm{i}}+\mathrm{a}_{\mathrm{ji}}+\mathrm{r}_{\mathrm{k}}+\mathrm{i} 1_{\mathrm{ik}}+\mathrm{i} 2_{\mathrm{jik}}+\mathrm{t}_{\mathrm{l}}+\mathrm{v}_{\mathrm{m}}+\mathrm{i} 3_{\mathrm{il}}+ \\
\mathrm{i} 4_{\mathrm{im}}+\mathrm{i} 5_{\mathrm{lm}}+\mathrm{b}_{1} \mathrm{E}_{\mathrm{v}}+\varepsilon_{\mathrm{ijklmn}}
\end{gathered}
$$

em que $Y_{\text {ijklmn }}=$ temperatura retal, temperatura da superfície da epiderme, temperatura interna do velo ou temperatura da superfície do velo observada no $n$-ésimo animal; $\mathrm{s}_{\mathrm{i}}=$ efeito fixo do $i$-ésimo sexo (1 e 2); $\mathrm{a}_{\mathrm{ji}}=$ efeito aleatório do $j$-ésimo animal pertencente ao $i$-ésimo sexo; $\mathrm{r}_{\mathrm{k}}=$ efeito fixo da $k$-ésima repetição $(1, \ldots, 5) ; i 1_{\mathrm{ik}}=$ efeito da interação $i$-ésimo sexo $\times$ k-ésima repetição; $\mathrm{i} 2_{\mathrm{jik}}=$ efeito da interação $j$-ésimo animal pertencente ao $i$-ésimo sexo $\times k$-ésima repetição; $\mathrm{t}_{\mathrm{l}}=$ efeito fixo da $l$-ésima temperatura do ar (25, 30 e $\left.40^{\circ} \mathrm{C}\right) ; \mathrm{v}_{\mathrm{m}}=$ efeito fixo da $m$-ésima movimentação do ar $(0$ e $5,0 \mathrm{~m} / \mathrm{s}) ; \mathrm{i} 3_{\mathrm{il}}=$ efeito da interação do $i$-ésimo sexo $\times$ l-ésima temperatura do ar; $i 4_{\text {im }}=$ efeito da interação $i$-ésimo sexo × m-ésima movimentação do ar; $i 5_{\mathrm{lm}}=$ efeito da interação l-ésima temperatura do ar × m-ésima movimentação do ar; $\mathrm{b}_{1}=$ coeficiente de regressão linear da característica observada em relação à espessura do velo; $\varepsilon_{\mathrm{ijklmn}}=$ resíduo, incluindo o erro aleatório; e $\alpha=$ intercepto. A média geral foi estimada por:

$$
\mu=\alpha+b_{1} \bar{E}
$$

em que $\overline{\mathrm{E}}=$ média geral da variável analisada.

As médias das variáveis estudadas foram comparadas pelo teste Tukey a $5 \%$ de probabilidade utilizando-se o 
programa estatístico “Statistical Analyses System” (SAS, 2001).

\section{Resultados e Discussão}

A análise de variância comprovou efeito significativo $(\mathrm{P}<0,05)$ da temperatura do ar para todas as variáveis estudadas (Figura1). O mesmo não foi observado para a movimentação do ar, que teve efeito apenas sobre a temperatura da epiderme (Figura 1). A interação temperatura $\times$ movimentação do ar foi significativa $(P<0,05)$ somente para temperatura da superfície do velo (Figura 2).

As médias das temperaturas reta, da epiderme e do interior do velo (Tabela 1) não sofreram alteração em maior movimentação de ar (Figura 1). No entanto, essas variáveis acompanharam a elevação da temperatura do ar, mas em escalas diferentes, de modo que a temperatura do interior do velo foi fortemente influenciada, enquanto as temperaturas retal e da epiderme foram com menor intensidade (Figura 1).
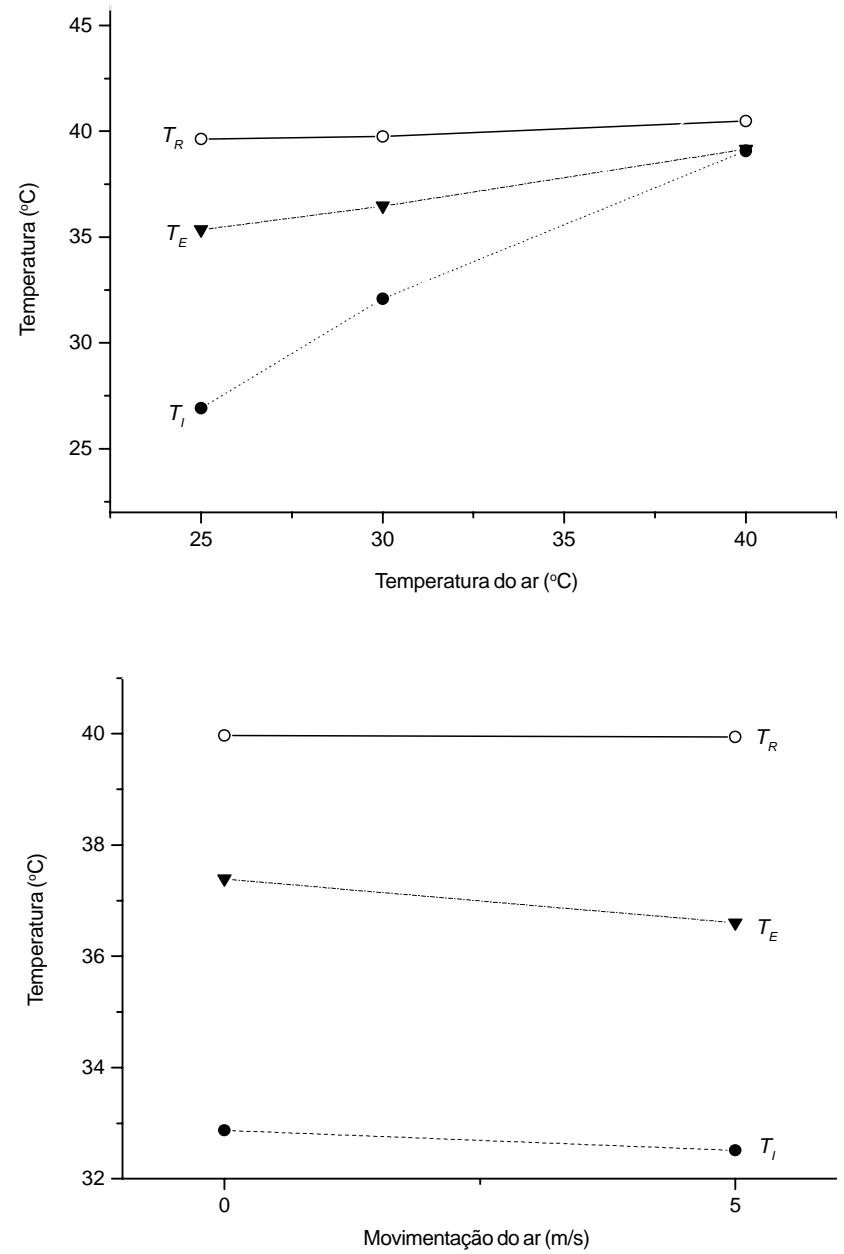

Figura 1 - Efeito da movimentação do ar sobre as temperaturas da epiderme $\left(T_{E}\right)$, do interior do velo $\left(T_{I}\right)$ e retal $\left(T_{R}\right)$.
Os resultados sugerem que o fluxo de ar $<5,0 \mathrm{~m} / \mathrm{s}$ e paralelo ao eixo do corpo do animal tem pouco efeito sobre o isolamento térmico, provavelmente porque a movimentação do ar sobre a superfície do velo não é eficiente em penetrar no seu interior e deslocar o ar preso entre as fibras, o qual determina a resistência térmica do velo e favorece a transferência de calor por convecção forçada. Resultados similares foram observados por Cena \& Monteith (1975b) em amostras de velo nas quais a condutividade térmica não sofria variações sob velocidades do vento entre 0 e $6,0 \mathrm{~m} / \mathrm{s}$, com fluxo paralelo à superfície do corpo.

Tabela 1 - Médias estimadas por quadrados mínimos, conforme a temperatura e movimentação do ar

\begin{tabular}{|c|c|c|c|}
\hline \multirow[t]{2}{*}{ Efeito } & \multicolumn{3}{|c|}{ Temperatura } \\
\hline & Retal & Superfície da epiderme & Interna do velo \\
\hline & \multicolumn{3}{|c|}{ Temperatura do ar $\left({ }^{\circ} \mathrm{C}\right)$} \\
\hline 25 & $39,63 c \pm 0,032$ & $35,35 c \pm 0,102$ & $26,91 c \pm 0,126$ \\
\hline 30 & $39,76 b \pm 0,032$ & $36,46 b \pm 0,103$ & $32,09 b \pm 0,128$ \\
\hline 40 & $40,48 a \pm 0,031$ & $39,16 a \pm 0,099$ & $39,08 \mathrm{a} \pm 0,123$ \\
\hline \multicolumn{4}{|c|}{ Movimentação do ar (m/s) } \\
\hline 0 & $39,97 a \pm 0,025$ & $37,39 a \pm 0,081$ & $32,77 \mathrm{a} \pm 0,120$ \\
\hline 5 & $39,94 a \pm 0,026$ & $36,60 \mathrm{~b} \pm 0,085$ & $32,55 a \pm 0,125$ \\
\hline
\end{tabular}

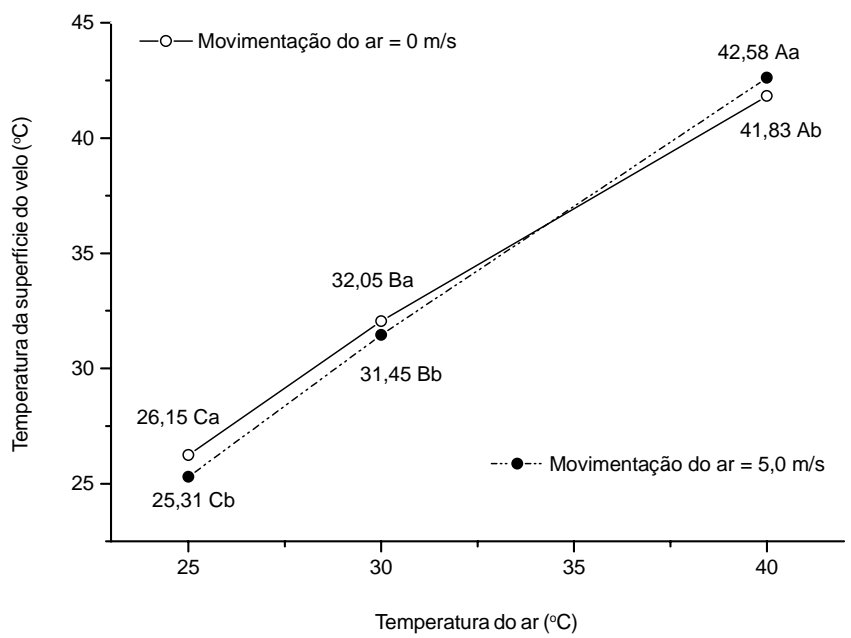

Figura 2 - Efeitos da interação entre temperatura e movimentação do ar sobre a temperatura da superfície do velo. Médias seguidas de letras diferentes, maiúsculas e na mesma linha, diferem estatisticamente entre si $(\mathrm{P}<0,05)$ pelo teste Tukey, enquanto médias seguidas de letras diferentes, minúsculas, entre linhas e para mesma temperatura do ar, diferem estatisticamente entre si $(\mathrm{P}<0,05)$ pelo teste Tukey. 
Esse fato poderia ser ocasionado pela orientação do animal em relação ao fluxo de ar, no entanto, Cena \& Monteith (1975b) observaram que a orientação do corpo em relação ao vento teve pouco efeito sobre a resistência média do velo. Por outro lado, de acordo com resultados obtidos por Blaxter et al. (1959), o isolamento térmico do velo é proporcional à sua espessura, assim, as hipóteses para o fraco desempenho da movimentação do ar em quebrar a resistência térmica do velo nesses animais seriam sua espessura, de 9,24 $\pm 0,082 \mathrm{~cm}$, e também o arranjo das fibras (Whates \& Charles, 1994), uma vez que velocidades do vento menores que $5,0 \mathrm{~m} / \mathrm{s}$ foram suficientes para reduzir metade do isolamento térmico em velos de até $5,0 \mathrm{~cm}$ de espessura.

O efeito da interação temperatura $\times$ movimentação do ar foi significativo $(\mathrm{P}<0,05)$ somente para temperatura da superfície do velo. Quando a movimentação do ar foi $5,0 \mathrm{~m} / \mathrm{s}$, houve redução significativa $(\mathrm{P}<0,05)$ na temperatura da superfície do velo de $26,15^{\circ} \mathrm{C}$ para $25,31^{\circ} \mathrm{C}$ quando a temperatura do ar era de $25^{\circ} \mathrm{C}$. A combinação temperatura do ar igual a $40^{\circ} \mathrm{C}$ e movimentação do ar igual a $5,0 \mathrm{~m} / \mathrm{s}$, no entanto, causou elevação significativa $(P<0,05)$ na temperatura da superfície do velo de 41,83 para $42,58^{\circ} \mathrm{C}$ (Figura 3). Obviamente, quando a temperatura do ar foi inferior a $30^{\circ} \mathrm{C}$, a movimentação do ar reduziu a temperatura da superfície do velo, fato que pode ser resultado da remoção do ar quente sob a superfície do velo oriundo do interior do mesmo, mas quando a temperatura do ar foi

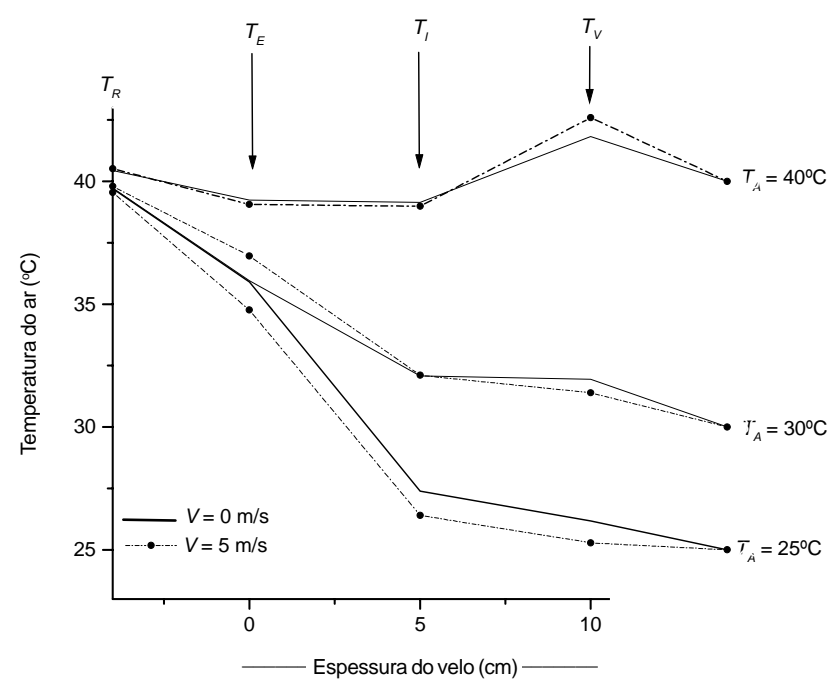

Figura 3 - Efeito da combinação da movimentação do ar (V) com a temperatura do ar sobre as temperaturas da epiderme $\left(T_{E}\right)$, do interior do velo $\left(T_{I}\right)$, da superfície do velo $\left(T_{V}\right)$ e retal $\left(T_{R}\right)$. superior a $40^{\circ} \mathrm{C}$ a movimentação do ar elevou a temperatura da superfície do velo, certamente por soprar sobre essa superfície a massa de ar quente proveniente do ambiente.

Em temperatura ambiente inferior a $30^{\circ} \mathrm{C}$, a transferência de calor através do velo foi dominada pela condução através das fibras e pela convecção livre pela movimentação do ar aprisionado entre as fibras (Figura 3), movimentação induzida por gradiente térmico causando diferença de densidade do ar no interior do velo, uma vez que a movimentação do ar falhou em penetrar através do velo. Esses resultados confirmam os relatados por Cena \& Monteith (1975b).

A diferença entre a temperatura de epiderme e a temperatura do ar foi de $-0,84^{\circ} \mathrm{C}$ quando a temperatura do ar foi $40^{\circ} \mathrm{C}$ (Tabela 1; Figura 3). Nesta condição, o fluxo de calor sensível foi negligível, indicando que os animais dependem somente do fluxo de calor latente para manter sua termorregulação. Silva \& Starling (2003) estimaram média de 48,14 W/m² para evaporação na superfície corporal e de $27,24 \mathrm{~W} / \mathrm{m}^{2}$ para evaporação respiratória em ovinos da raça Corriedale do mesmo rebanho submetidos às condições de temperaturas do ar similares às utilizadas neste trabalho. O fluxo de calor latente de aproximadamente $75,38 \mathrm{~W} / \mathrm{m}^{2}$ estimado por esses autores parece não ser suficiente para a manutenção da temperatura corporal estável (Figuras 1 e 3) nesses animais, uma vez que ocorreu elevação da temperatura retal quando a temperatura do ar foi superior a $40^{\circ} \mathrm{C}$, certamente em razão da estocagem de energia térmica (McLean et al., 1983).

As temperaturas da epiderme e retal foram significativamente $(\mathrm{P}<0,05)$ correlacionadas somente com temperatura do ar, e não com a movimentação do ar $(\mathrm{m} / \mathrm{s})$ e a correlação com a temperatura de epiderme foi igual a 0,81 e com temperatura retal, igual a 0,62 (Figuras 1 e 2). No entanto, a temperatura da superfície do velo foi significativamente $(\mathrm{P}<0,05)$ correlacionada com a temperatura do $\operatorname{ar}(r=0,98)$, mas a analise de variância mostrou que o efeito da interação entre temperatura do ar e movimentação do ar foi significativa $(\mathrm{P}<0,05)$. Este resultado indica que a temperatura de superfície do velo varia com a temperatura e movimentação do ar. Assim, a temperatura de superfície do velo deve ser estimada por uma função que considere tanto a temperatura como a movimentação do ar, que foi dada pela equação de regressão linear múltipla $T_{V}=0,2556+1,044 T_{A}+0,035 V$, com R ${ }^{2}=0,97$.

\section{Conclusões}

A movimentação do ar, natural ou artificial, com o objetivo de favorecer a perda de calor sensível através do 
velo em ovinos não é eficiente para o isolamento térmico e para favorecer a transferência de calor sensível do interior para superfície externa em velos com espessura média de $9,0 \mathrm{~cm}$.

\section{Literatura Citada}

BERMAN, A. Tissue and external insulation estimates and their effects on prediction of energy requirements and of heat stress. Journal of Dairy Science, v.87, p.1400-1412, 2004.

BLAXTER, K.L.; GRAHAM, MEC, N.; WAINMAN, F.W. Environmental temperature, energy metabolism and heat regulation in sheep. III. The metabolism on thermal exchange of sheep with fleeces. Journal Agricultural Science, v.52, p.41-49, 1959.

CENA, K.; MONTEITH, J.L. Transfer processes in animal coats. I. Radiative transfer. Proceedings of the Royal of Society London, B Biological Sciences, v.188, n.1, p.377-393, 1975a.

CENA, K.; MONTEITH, J.L. Transfer processes in animal coats. II. Conduction and convection. Proceedings of the Royal of Society London, B Biological Sciences v.88, n.1, p.395-411, 1975b.

CENA, K.; MONTEITH, J.L. Transfer processes in animal coats. III. Water vapor diffusion. Proceedings of the Royal of Society London, B Biological Sciences, v.188, n.1, p.413423, 1975c.

DAVIS, L.B.; BIRKEBAK, R.C. On the transfer of energy in layers of fur. Biophysical Journal, v.14, p.249-268, 1974.

EHRLEMARK, A.G.; SALLVIK, K.G. A model of heat and moisture dissipation from cattle based on thermal properties. Transactions of the ASAE, v.39, n.1, p.187-194, 1996.
GEBREMEDHIN, K.G.; PORTER, W.P.; CRAMER, C.O Quantitative analysis of the heat exchange through the fur layer of Holstein calves. Transactions of the ASAE, v.26, n.1, p.188-193, 1983.

LITTELL, R.C.; FREUND, R.J.; SPECTOR, P.C. SAS ${ }^{\circledR}$ system for linear models. 3.ed. Cary: SAS Institute, 1991. 329p.

McARTHUR, A.J.; MONTEITH, J.L. Air movement and heat loss from sheep. II. Thermal insulation of fleece in wind. Proceedings of the Royal of Society London, B Biological Sciences, v.209, p.209-217, 1980.

McARTHUR, A.J. Thermal interaction between animal and microclimate: a comprehensive model. Journal Theorical Biology, v.126, p.203-238, 1987.

McLEAN, J.A.; STOMBAUGH, D.P.; DOWNIE, A.J. Body heat storage in steers (Bos taurus) in fluctiating thermal environments. Journal Agricultural Science, v.100, p.315322, 1983.

MONTEITH, J.L.; UNSWORTH, M.H. Principles of environmental physics. 2.ed. London: E. Arnold, 1990. 291p.

STATISTICAL ANALYSES SYSTEM - SAS. SAS user's guide: statistics. Cary: 2001. 956p.

SILVA, R.G.; STARLING, J.M.C. Evaporação cutânea e respiratória em ovinos sob altas temperaturas ambientes. Revista Brasileira de Zootecnia, v.32, n.8, p.1956-1961, 2003.

TREGGEAR, R.T. Hair density, wind speed and heat loss in mammals. Journal of Applied Physiology, v.20, p.796-801, 1965.

WALSBERG, G.E.; CAMPBELL, G.S.; KING, J.R. Animal coat color and relative heat gains: a re-evatuation. Journal Comparative Physiology, v.126, p.211-222, 1978.

WHATES, C.M.; CHARLES, D.R. Livestock housing. Wallingford: CAB International, 1994. p.37-60. 